\title{
Ordre des constituants en chinois ancien : Cas particulier de la place du pronom objet dans les phrases négatives
}

\author{
Redouane Djamouri*
}

\section{INTRODUCTION}

Le chinois archaïque est une langue à ordre (S)VO qui n'a pour adpositions que des prépositions et dont l'ordre de détermination au sein du syntagme nominal est toujours 'déterminant-déterminé'. Ces caractéristiques ne semblent plus aujourd'hui devoir être remises en doute. Contrairement à ce que des linguistes comme Li et Thompson (1974) ont pu laisser croire, les plus anciens documents découverts à ce jour, à savoir les inscriptions sur os et carapaces de tortue de l'époque Shang (13e-11e s. av. J.-C.), témoignent déjà d'un ordre SVO. Cette dernière observation est confirmée par l'ensemble des études de première main consacrées à ces documents (cf. Guan Xiechu 1953; Chen Mengjia 1956 ; Serruys 1974, 1981 ; Takashima 1973, 1990 ; Kryukov 1980 ; Djamouri 1988 ; Djamouri \& Paul 1997 ; Shen Pei 1992, Zhang Yujin 2001 etc.).

Nous nous intéresserons ici à une structure particulière du chinois ancien, à savoir la position préverbale du pronom objet dans des phrases négatives. Ce phénomène syntaxique est observable sur plusieurs siècles, des plus anciennes inscriptions (époque Shang, $13^{\mathrm{e}}-11^{\mathrm{e}} \mathrm{s}$. av. J.-C.) jusqu'aux textes de la fin de la dynastie des Han ( $2^{\mathrm{e}} \mathrm{s}$.). L'exemple ci-dessous, datant de l'époque Shang, reprend deux énoncés parallèles faisant partie d'une même inscription. Le premier énoncé, de forme affirmative, témoigne d'un ordre VO où le pronom objet est en position postverbale (ex. (1a)); le second, de forme négative, voit le même pronom objet apparaître en position préverbale, entre la négation et le verbe (ex. (1b)) :

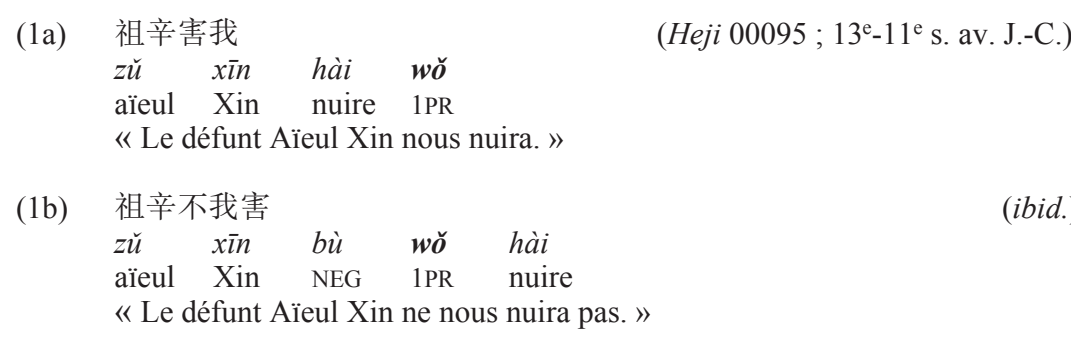

\footnotetext{
* CNRS-EHESS-INALCO, Centre de Recherches Linguistiques sur l'Asie Orientale. Courriel : djamouri@ehess.fr.
} 
La plus ancienne observation de ce phénomène est celle qu'un lettré des Tang, Kong Yingda (574-648), fait dans son commentaire du Livre des Odes. Il se contente d'indiquer que l'on a là un des nombreux cas d'inversions attestées en chinois archaïque.

On doit à Abel Rémusat une des toutes premières tentatives d'explication de ce phénomène. Dans la grammaire qu'il publie en 1857 (p. 66-67), il y voit une inversion stylistique qu'il formule en ces termes : «Les pronoms, complémens d'un verbe actif, font quelquefois exception à la règle précédente, par l'effet d'une inversion élégante; ainsi l'on trouve 不吾知也 'il ne me connoît pas' au lieu de 不知吾也 qui serait plus régulier. »

Julien $(1869: 147-149)$ et Von der Gabelentz (1881:145-149) consacrent, 1'un comme l'autre, un court chapitre à 'l'antéposition'. Tous deux mentionnent le phénomène qui nous intéresse mais n'en donnent pas d'explication. Von der Gabelentz formule son incompréhension en ces termes : «L'antéposition, qui ne concerne quasiment jamais des substantifs ou des noms propres, bouleverse l'ordre de la phrase de manière curieuse et difficilement explicable ».

Ma Jianzhong (1898), dans la première grammaire chinoise d'inspiration occidentale consacrée à la langue archaïque, constate seulement que la position préverbale est la position « habituelle » du pronom objet dans une phrase négative. Bien plus tard, se penchant sur le phénomène en question, Wang Li (1958) précise que l'on ne saurait y voir une phrase où l'ordre des constituants serait inversé (dăozhuàng jù 倒裝句) dans la mesure où il s'agit là de l'ordre par défaut propre à ce contexte syntaxique, sans autre position possible pour le pronom objet.

Zhou Guangwu (1959), dans le premier article entièrement consacré à ce phénomène, montre, données à l'appui, que dans les documents antérieurs aux Qin (221 av. J.-C.) le pronom objet dans les phrases négatives apparaît à la fois en position préverbale mais aussi en position postverbale, dans des proportions équivalentes.

De même, sur la base du décompte des positions pré et postverbales du pronom objet dans les phrases négatives effectué par Tang (1990), Peyraube (1997) conclut que « l'ordre est relativement libre, sans indication décisive sur le fait que l'un des deux ordres est plus fondamental que l'autre $»$.

Il convient également de mentionner, entre autres travaux conduits dans un cadre générativiste, l'étude de Feng (1996) proposant une motivation d'ordre prosodique qui aurait donné lieu à une cliticisation du pronom par son adjonction à droite de la négation dominant le syntagme verbal, ainsi que l'étude récente d'Aldridge (2015) qui considère que « the object was forced to undergo object shift to the edge of $n \mathrm{P}$. Since structural case was unavailable, the object was forced to undergo object shift to the edge of $n \mathrm{P}$, where it could exceptionally value accusative case with the head of NegP ». Ces analyses ne rendent pas compte (ou imparfaitement) des cas où, dans des phrases négatives, l'objet pronominal reste en position postverbale.

Nous insisterons ici sur le fait que la position préverbale du pronom objet en chinois ancien ne peut être considérée comme un reliquat d'un ordre OV dans une langue qui, à l'ère historique, serait devenue VO (cf. Djamouri 2000, 2001, 2014 ; Djamouri, Paul \& Whitman 2013 ; Meisterernst 2010, Aldridge 2015). 
Nous tenterons de montrer que l'emploi du pronom objet en position préverbale dans les phrases négatives répond à une contrainte syntaxique qui ne s'est exercée de manière stricte qu'à la période la plus ancienne, celle des inscriptions Shang. Les documents ultérieurs, quant à eux, contiennent presque autant de phrases négatives où le pronom objet apparaît en position préverbale que de phrases négatives où il apparaît en position postverbale.

Nous essaierons par ailleurs d'apporter un nouvel éclairage consistant à prendre en compte le statut des propositions dans lesquelles l'un ou l'autre positionnement est observé.

\section{Position de L'OBJET DANS LES INSCRIPTIONS SHANG $\left(13^{\mathrm{E}}-11^{\mathrm{E}}\right.$ S. AV. J.-C. $)$}

\subsection{Objet nominal}

Sur la base d'un corpus de plus de 26000 énoncés complets, il nous a été possible de relever $94 \%$ d'énoncés $(\mathrm{S}) \mathrm{VO}$ pour seulement $6 \%$ d'énoncés $(\mathrm{S}) \mathrm{OV}$. Ce décompte ne concerne que les compléments véritables, ou arguments, et non les circonstants ${ }^{1}$. Dans un énoncé non-marqué, tout complément de statut argumental apparaît en position postverbale, qu'il soit représenté par un syntagme nominal ou par un syntagme prépositionnel, qu'il corresponde à un patient (2), un destinataire (3) ou un locatif (4).

(2) 王伐台方

wáng fá $\quad$ gōng fāng
roi combattre Gong tribu
«Le roi combattra la tribu Gong.

(Heji 622)

« Le roi combattra la tribu Gong. »

(3) 帝其受我佑

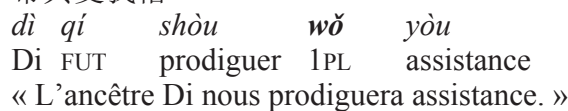

(4) 我乎往于西

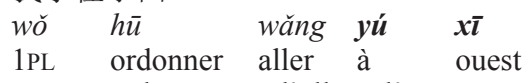

(Heji 07440 verso)

« Nous ordonnerons d'aller à l'ouest. »

En revanche, les énoncés témoignant d'un ordre (S)OV (6\% du total) sont des énoncés marqués, confirmant en cela la généralisation formulée par Hagège (1975: 214) : « dans une langue à séquence prédicat+objet, la position pré-prédicative est une position remarquable, c'est à dire exploitable aussi bien pour focaliser que pour exprimer l'objet non virtuel, la focalisation étant également actualisante ».

Deux types d'énoncés SOV sont à distinguer: $1^{\circ}$ Les énoncés où l'objet

\footnotetext{
${ }^{1}$ Les circonstants sont, à cette époque, plus libres dans leur distribution. Ils apparaissent en position pré ou postverbale sans distinction apparente (cf. Djamouri \& Paul 1997).
} 
préverbal (qu'il s'agisse d'un syntagme nominal ou d'un syntagme prépositionnel) est focalisé au moyen d'une construction clivée formée à l'aide d'une copule (ex. $(5 \mathrm{a}, \mathrm{b})) ; 2^{\circ}$ Les énoncés négatifs où le pronom personnel objet apparaît en position préverbale $(1 \mathrm{~b})$; ces derniers sont précisément ceux qui retiendront notre attention dans la suite de cet article.

(5a) 王勿唯龍方伐
wáng wù wéi lóng fāng fá
roi NEG.devoir être Long tribu combattre
« Ce ne doit pas être la tribu Long que le roi combattra. »

(Heji 6476)

(5b) 王惠龍方伐

$\begin{array}{llll}\text { wáng huì } & \text { lóng fāng fá } \\ \text { roi } & \text { devoir.être } & \text { Long } & \text { tribu combattre }\end{array}$

« Ce doit être la tribu Long que le roi combattra.»

On soulignera que dans les deux phrases clivées citées en (5) l'objet focalisé est complément de la copule. La séquence ainsi formée [COP NOM.OBJ] se trouve en parfaite adéquation avec l'ordre VO qui, jusqu'à nos jours, caractérise la langue chinoise.

\subsection{Objet pronominal}

Les données de la période archaïque, prises dans leur totalité, sont relativement complexes. On ne dénombre pas moins de vingt formes pronominales et neuf négations ; par ailleurs, comme nous le verrons plus bas, la position du pronom objet dans une phrase négative n'a pas toujours été exclusivement préverbale. À cet égard, les inscriptions Shang présentent un tableau relativement simple : il n'existe pas encore de pronom personnel de troisième personne (rôle que remplissaient alors noms ou pronoms démonstratifs); les pronoms objets de 1 ère et $2^{\mathrm{e}}$ personnes apparaissent en position postverbale dans les phrases affirmatives (cf. ex. (1a)) et préverbale dans les phrases négatives (cf. ex. (1b)).

Il est crucial de noter que la position préverbale du pronom-objet est alors toujours liée à la présence de la négation bù 不. Pour expliquer ce phénomène, il convient avant tout d'examiner la différence entre l'emploi de bì et celui d'une autre négation, $f^{\prime}$ 弗. La distinction fondamentale entre ces négations est que l'une, bù, est utilisée pour nier des verbes intransitifs (ex. (6), (7) et (8)), et que l'autre, $f u ́$, ne nie que des verbes transitifs (ex. (9) et (10)):

(6)

$$
\begin{aligned}
& \text { 不雨 } \\
& \text { bù } y \check{u} \\
& \text { NEG pleuvoir } \\
& \text { «Il ne pleuvra pas. » }
\end{aligned}
$$

(7) 王不往田

$$
\begin{array}{llll}
\text { wáng } & \text { bù } & \text { wăng } & \text { tián } \\
\text { roi } & \text { NEG } & \text { aller chasser }
\end{array}
$$$$
\text { « Le roi n'ira pas chasser. » }
$$ 
（8）生十三月婦好不其來 (Heji 2653) shēng shí sān yuè fù hăo bù qi lái commençant dix trois mois Dame Hao NEG FUT venir « Au début du treizième mois, Dame Hao ne reviendra pas (de son expédition). »

(9) 黃尹弗害王

huángyı̀n fú hài wáng

Huangyin NEG nuire roi

« L'ancêtre Huangyin ne nuira pas au roi. »

(10) 台方弗敦沚

gong fāng fú dūn zhĭ

Gong tribu NEG attaquer Zhi

« La tribu Gong n'attaquera pas (l'officier) Zhi. »

(Heji 6946 recto)

On relèvera par ailleurs que certains verbes transitifs suivis de leur objet sont niés aussi bien par fú que par bù. Cela n'est néanmoins possible qu'avec des verbes de transitivité faible, où l'objet (en tant que patient) reste extérieur au procès et ne subit pas de modification de son état. C'est notamment le cas avec des verbes comme jiàng 降 'envoyer' (ex. (11)), gòu 遘 'rencontrer' (ex. (12)), shòu 受 'recevoir' (ex. (13)), ou bin 賓 'honorer' etc. :

(11a) 帝弗其降禍

dì fú qi jiàng huò

(Heji 14176)

Di NEG FUT envoyer malheur

«(Le dieu) Di ne nous enverra pas de malheur. »

(11b) 帝不其降熯

dì bù qi jiàng hàn

(Zongtu 22.7)

Di NEG FUT envoyer sécheresse

« Di ne nous enverra pas de sécheresse. »

(12a) 甫弗遘台方

fǔ fú gòu gōng fāng

$\mathrm{Fu}$ NEG rencontrer Gong tribu

«Fu ne rencontrera pas (des gens de) la tribu Gong. »

(12b) 戊不其遘戎

yuè bù qi gòu róng

Yue NEG FUT rencontrer Rong

" (Le commandant) Yue ne rencontrera pas (des gens de) la tribu Rong.'

(13a) 我弗其受黍年

wǒ fú qi shòu shǔ nián

(Heji 9950 recto)

1PL NEG FUT recevoir millet récolte

« Nous ne bénéficierons pas d'une (bonne) récolte de millet. » 
(13b) 我不其受㯟年
wǒ bù $q \grave{u}$
qi shòu shǔ nián
1 L

(Heji 9956)

« Nous bénéficierons d'une (bonne) récolte de millet. »

On notera enfin qu'il est des cas où la négation bù est utilisée pour nier des verbes transitifs forts (impliquant une modification de l'état du patient). Cet emploi révèle cependant un changement dans la relation entre le sujet et le verbe. Comme le montrent les exemples (14a) et (14b), un verbe employé transitivement est nié par fú, mais lorsqu'il est employé comme prédicat descriptif ou attributif ne pouvant régir de patient, il est alors nié par bù :

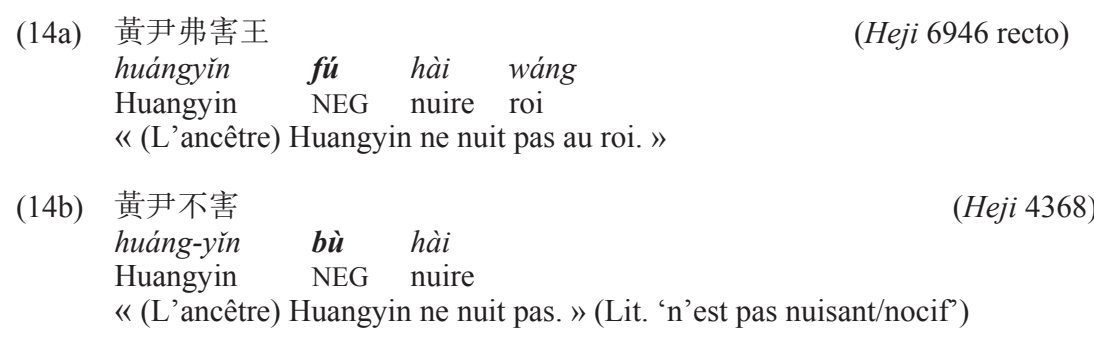

Il ressort de (14) que fú doit être vue comme une négation proprement adverbiale ('ne... pas'), alors que bù doit être interprétée comme une copule verbale de valeur négative ('ne pas être').

Cet emploi de bù doit être rapproché de celui des autres copules verbales présentes dans les inscriptions Shang (cf. wéi et hui dans l'ex. (5a,b)). Outre leur emploi dans des clivées, ces copules permettent de construire des prédicats nominaux ou adjectivaux dans des phrases simples (cf. Djamouri 2001).

L'emploi de bù en tant que copule apparaît nettement lorsque le patient est posé comme sujet syntaxique. On obtient alors une construction moyenne ou le verbe est employé comme prédicat de nature descriptive (à l'instar d'un participe en français). Les exemples (15) à (17) permettent de comparer deux à deux, pour un même verbe, un emploi transitif et actif nié par fú [S fú $\left.\mathrm{V} \mathrm{N}_{\text {Patient }}\right]$ et un emploi descriptif nié par bù où le patient est posé comme sujet d'un énoncé passif, nonagentif $\left[\mathrm{N}_{\text {Patient }}\right.$ bù $\left.\mathrm{V}\right]$ :
(15a) 今夕弗辰 王師

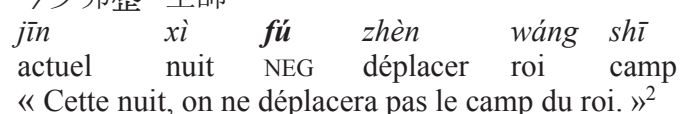

(Heji 36427)

\footnotetext{
${ }^{2}$ Noter que le sujet n'a jamais été une expansion obligatoire en chinois. L'énoncé, même en l'absence du sujet, reste ici actif.
} 


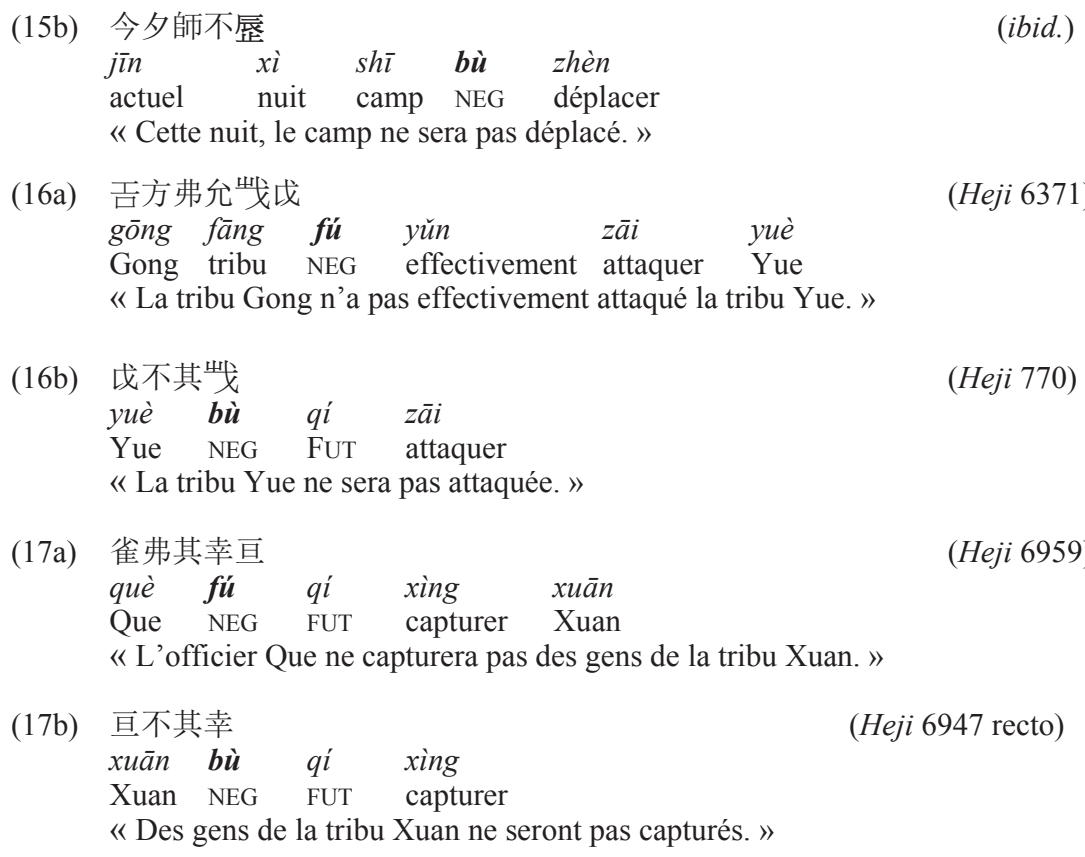

L'emploi de bù en tant que copule apparaît de manière plus évidente encore dans des phrases descriptives ayant pour prédicat un véritable adjectif (ex. (18)) ou un adjectif dénominal (ex. (19)-(20)). Dans l'exemple (18), on notera avec intérêt la présence en un même énoncé des deux négations fú et bù ; la première niant le prédicat verbal de la proposition-sujet, la seconde niant l'adjectif posé comme prédicat principal de l'énoncé :

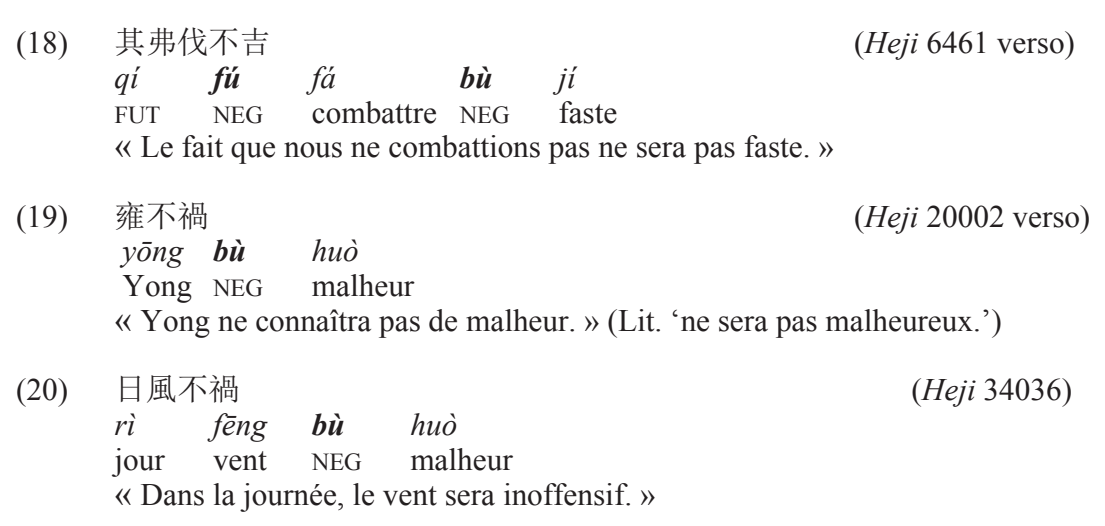


L'emploi de bù en tant que copule dans des énoncés descriptifs n'est pas le seul rapprochement fonctionnel qu'il est permis de faire avec les copules wéi ou hùi. Il existe des énoncés dans lesquels bù permet de former une clivée tout en focalisant un constituant en position préverbale. C'est le cas en (21) où l'on a deux énoncés parallèles faisant partie d'une même inscription. En (21b), bù est employée comme contrepartie négative de wéi (ex. (21b)) ; l'une comme l'autre forment ici une clivée permettant de focaliser l'agent (représenté ici par le syntagme nominal fù $Y_{l}$ 'Père défunt Yi').

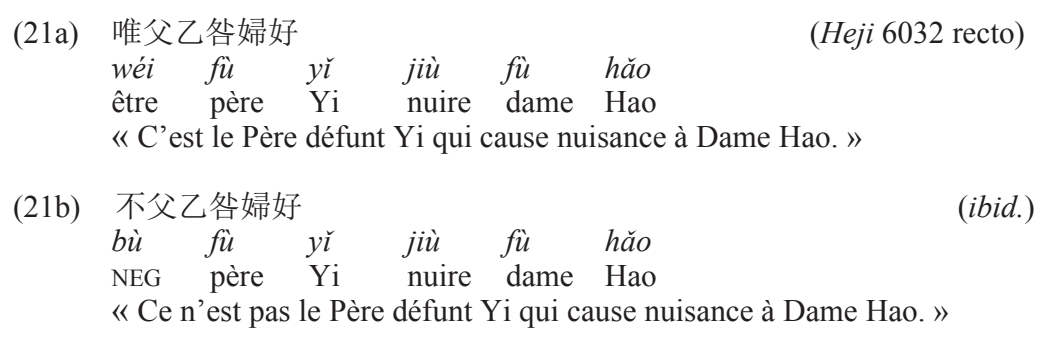

C'est précisément ce rôle de copule négative dans une phrase clivée qui permet de comprendre pourquoi bù se construit avec un pronom objet en position préverbale. Ainsi, lorsqu'un verbe transitif a pour objet un nom, l'ordre reste toujours VO, même dans une phrase négative (22) :

(22)

$$
\begin{aligned}
& \text { 父甲弗害王 } \\
& \text { fù jiă fú hài wáng } \\
& \text { père Jia NEG nuire roi } \\
& \text { « Le Père défunt Jia ne cause pas nuisance au roi. » }
\end{aligned}
$$

(Heji 2120)

De la même manière, dans une phrase affirmative, le pronom objet apparaît toujours en position postverbale (23):

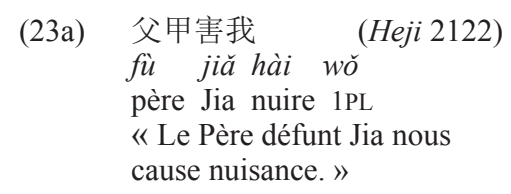
(23b) ＊ 父甲我害 fù jiă wǒ hài père Jia 1PL nuire

En revanche, la forme négative de (23a) ne peut être obtenue qu'en ayant recours à la négation bù avec laquelle le pronom objet peut se construire en position préverbale (24):

(24) 父甲不我害

$$
\begin{array}{lllll}
f \hat{u} & j i a ̆ & \text { bù } & \text { wǒ } & \text { hài } \\
\text { père } & \text { Jia } & \text { NEG } & \text { PL } & \text { nuire }
\end{array}
$$$$
\text { " Le Père défunt Jia ne nous cause pas nuisance. » }
$$ 
Que ce soit en position postverbale (25) ou préverbale (26), l'objet pronominal n’apparaît jamais dans un énoncé nié par fú:

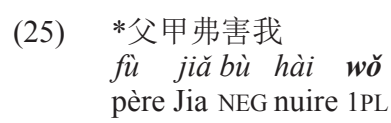
(26) * *父甲弗我害 fù jiăfú wŏ hài père Jia NEG 1PL nuire

En résumé : $1^{\circ}$ Dans les inscriptions Shang $\left(13^{\mathrm{e}}-11^{\mathrm{e}}\right.$ s. av. J.-C.), un objet argumental ne peut apparaître en position préverbale que s'il est focalisé dans une structure clivée à l'aide d'une copule (wéi 'être' ou hui 'devoir être') ; $2^{\circ} \mathrm{La}$ négation bù, fonctionnant également comme une copule ('ne pas être'), permet de construire une clivée à valeur focalisante (cf. ex. (21b)). De ces observations, nous pouvons légitimement déduire, qu'avant les Shang, la structure 'bù PrONObjet V' a dû correspondre à une clivée focalisant l'objet pronominal. Il est cependant important de rappeler qu'à l'époque Shang, la structure (24) s'impose comme la seule possible pour nier un verbe à objet pronominal ; elle aurait perdu l'effet focalisant qui en faisait une structure marquée.

Voyons maintenant de quelle manière ce phénomène s'est perpétué aux époques ultérieures.

\section{POSITION DU PRONOM OBJET DANS LES TEXTES ULTERIEURS}

\subsection{Données}

\subsubsection{Période des Zhou Occidentaux (11 $1^{e}-8^{e}$ s. av. J.-C.)}

Dans les inscriptions sur bronze des Zhou Occidentaux, nous n'avons qu'une seule occurrence de phrase négative à objet pronominal. Cette quasi-absence ne peut être imputée qu'à un manque de données paléographiques et non à une contrainte grammaticale. L'exemple en question (cf. ex. (27)) correspond à une phrase impérative où l'on a une négation de valeur prohibitive (wù 勿 'ne pas devoir'). Le pronom objet yú 余 est suivi d'une séquence appositive qui rend impossible son emploi préverbal (celui-ci conduirait à une interprétation pour le moins problématique du type : 'Ce ne doit pas être moi que tu surpasseras, ton unique souverain').

$$
\begin{aligned}
& \text { 女勿克余乃辟一人 bi (Yu ding) } \\
& \begin{array}{lllllll}
r \check{u} & w \grave{u} & k e ̀ & \boldsymbol{y u ́} & n a ̆ i & b \grave{i} & y \bar{\imath} \text { rén } \\
\text { 2SG } & \text { NEG } & \text { surpasser } & \text { 1SG } & \text { 2SG.POSS } & \text { souverain } & \text { un homme }
\end{array} \\
& \text { « Tu ne dois pas me surpasser, moi ton unique souverain. » }
\end{aligned}
$$

\subsubsection{Période des Zhou Orientaux ( $8^{e}-2^{e}$ s. av. J.-C.)}

Pour la période des Zhou Orientaux (allant de 771 jusqu'à la conquête des Qin 221 av. J.-C.), nous présentons (voir tableau I) une synthèse simplifiée et corrigée du décompte établi par Zhou Guangwu (1959). Nous avons là le nombre 
d'occurrences des pronoms objets en fonction des positions pré et postverbales qu'ils peuvent prendre et des différentes négations avec lesquelles ils apparaissent. Ce décompte partiel a été établi à partir de seize textes, parmi les plus importants de la période pré-Qin $\left(8^{\mathrm{e}}-2^{\mathrm{e}}\right.$ s. av. J.-C.) : Shangshu 尚書, Shijing 詩經, Lunyu 論 語, Zuozhuan 左傳, Guoyu 國語, Mengzi 孟子, Zhuangzi 莊子, Mozi 墨子, Xunzi 荀子, Hanfeizi 韓非子, Gongyangzhuan 公羊傳, Zhanguoci 戰國策, Quzi 曲子, Lüshi chunqiu 呂氏春秋, Guanzi 管子, and Yanzi Chunqiu 晏子春秋).

\begin{tabular}{|l|c|c|c|c|c|c|}
\hline & $\begin{array}{r}\text { Pronoms personnels de } \\
1^{e} \text { et } 2^{e} \text { personnes }\end{array}$ & \multicolumn{2}{|c|}{ Pronom objet zhī 之 } & \multicolumn{2}{c|}{ démonstratifs } \\
\cline { 2 - 7 } & préverbal & postverbal & préverbal & postverbal & préverbal & postverbal \\
\hline bù 不 & 117 & 96 & $1 ?$ & 206 & 13 & 72 \\
\hline$f u ́$ 弗 & 0 & 0 & 0 & 11 & 0 & 1 \\
\hline wù 勿 & 1 & 2 & 0 & 11 & 0 & 1 \\
\hline wú 冊/無 & 15 & 11 & 1 & 18 & 3 & 7 \\
\hline wèi 未 & 7 & 3 & 85 & 20 & 1 & 4 \\
\hline mò 莫 & 38 & 4 & 156 & 36 & 0 & 8 \\
\hline Total & $\mathbf{1 7 8}$ & $\mathbf{1 1 6}$ & $\mathbf{2 4 3}$ & $\mathbf{3 0 2}$ & $\mathbf{1 7}$ & $\mathbf{9 3}$ \\
\hline
\end{tabular}

Tableau I : occurrences et distribution, à l'époque pré-Qin, des pronoms objets dans des phrases négatives en fonction de différentes négations

Le tableau I appelle les commentaires suivants :

Selon sa catégorie et le type de négation avec laquelle il est employé, le pronom objet peut apparaître (majoritairement ou exclusivement) en position préverbale ou en position postverbale.

La majorité des pronoms objets de 1ère et de 2ème personne apparaissent en position préverbale ; ils sont le plus souvent employés avec la négation bù.

La négation fú n'est jamais associée à un pronom objet de première ou de deuxième personne. Dans les rares cas où elle est employée avec le pronom objet de troisième personne $z h \bar{\imath}$ ou avec un pronom démonstratif objet, ces derniers sont toujours en position postverbale. Ding (1935) a émis l'hypothèse que fú était le résultat d'une coalescence entre la négation bù et le pronom objet de troisième personne $z h \bar{l}$. Nombre d'auteurs ont par la suite repris cette hypothèse sans toutefois examiner les données empiriques à même de la confirmer (cf. inter alia Boodberg 1937, Lü 1942, Wang 1958 (qui considère que l'on a là, non pas une coalescence phonétique, mais un amalgame fonctionnel de la négation et du pronom objet $z h \bar{l})$, Kennedy 1952, Graham 1952 et Mulder 1959). Plus près de nous, He (2001) a pu montrer que l'hypothèse en question n'est pas corroborée par les données textuelles antérieures aux Qin ( $3^{\mathrm{e}} \mathrm{s}$. av. J.-C.) où l'on trouve nombre de cas où le pronom objet de troisième personne $z h \bar{\imath}$ apparaît après un verbe nié par fú.

Le pronom objet de troisième personne $z h \bar{l}$ est employé avec toutes les négations et apparaît le plus souvent en position postverbale, sauf avec wèi et mò avec lesquelles il apparaît très majoritairement en position préverbale.

Tel qu'il se présente, le tableau I fait ressortir nombre de dissymétries mais ne 
permet guère de différencier les emplois préverbaux des pronoms objets de leurs emplois postverbaux. L'explication linguistique que nous proposons ci-après tient compte d'une contrainte syntaxique liée au statut des propositions (sur ce point, voir Djamouri 2000, 2014).

\subsection{Statut propositionnel et position du pronom objet}

\subsubsection{Position préverbale}

De manière régulière, lorsque la proposition négative est une proposition finie, le pronom objet apparaît en position préverbale. Il peut s'agir d'une proposition indépendante, d'une proposition principale ou d'une véritable proposition subordonnée ; dans tous ces cas, les verbes de ces propositions reçoivent, ne seraitce que dans leur interprétation, toutes les spécifications temporelles et aspectuelles nécessaires à leur autonomie en tant que prédicats principaux ou secondaires.

C'est ainsi que l'on retrouve dans les propositions indépendantes l'ordre [NEG PrON.OBJ V] (cf. ex. (28)-(39)). On notera cependant que cette structure n'est plus liée au seul emploi de négation bù comme à la période Shang, mais qu'elle s'est généralisée à l'ensemble des négations. Une fois encore, le fait que l'on ait là la seule structure possible pour ce type de proposition ne permet pas d'y voir une structure marquée.

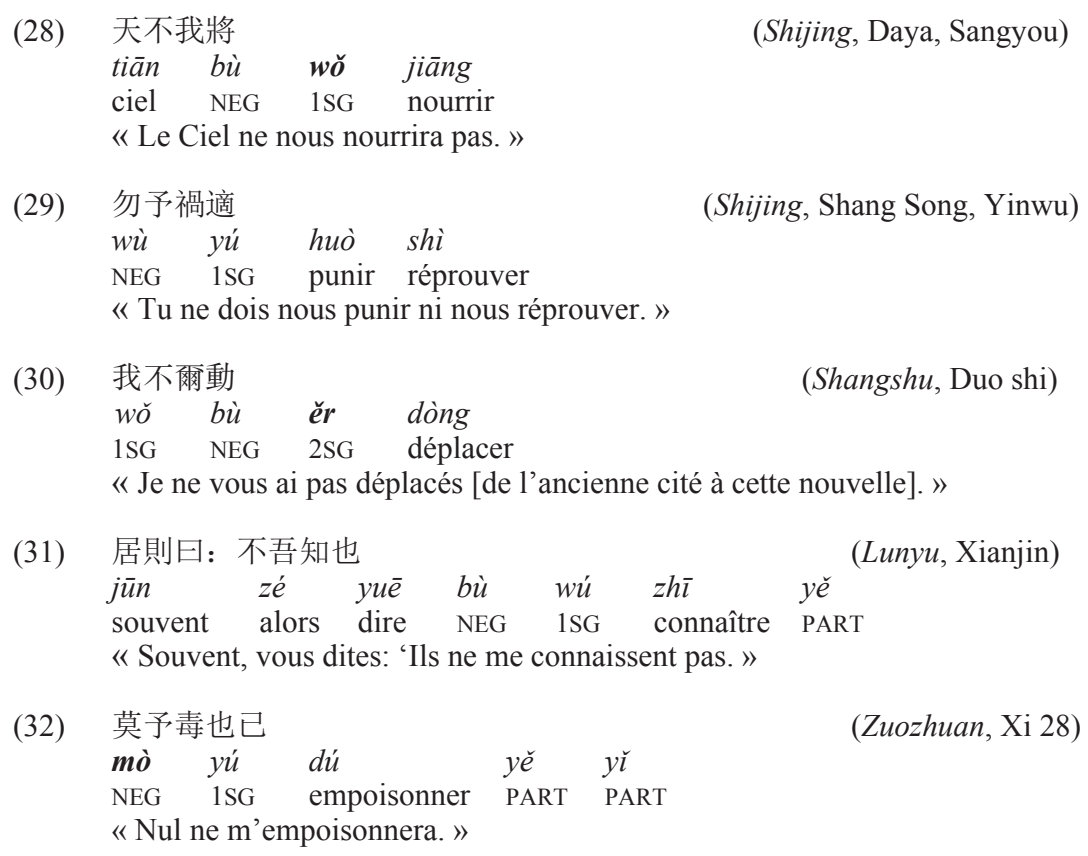


(33) 吾先君亦莫之行也

$\begin{array}{llllllll}\text { wú } & x i \bar{a} n & j \bar{u} n & y \grave{i} & \boldsymbol{m} \grave{o} & z h \bar{\imath} & \text { xíng } & y \check{~} \\ 1 \mathrm{SG} & \text { précédent } & \text { souverain } & \text { aussi } & \text { NEG } & 3 & \text { mener } & \text { PART }\end{array}$

«De même, parmi les précédents souverains, il n'y en a eu aucun qui le mena [un tel deuil]. » (Mengzi, Teng 1)

(34) 古今莫之能廢也
$g \check{u} \quad j \bar{\imath} n$
fèi pouvoir
se.passer.de PART
« De tout temps nul n'a pu s'en passer [d'une armée]. » (Guanzi, 16, 3/14)

Mò, employée dans les exemples (32)-(34), est une négation complexe qui, outre sa valeur négative, tient lieu de pronom indéfini au rôle agentif (équivalent au fr. 'nul ne...', 'il n'y a personne qui ne...'). La séquence [mò PrON.OBJ V] ne saurait d'aucune manière être analysée comme une clivée (avec ou sans interprétation focalisante). La position préverbale du pronom objet ne fait que répondre à la distribution par défaut qui doit être la sienne en tant que négation d'un prédicat verbal dans une proposition indépendante. Cette dernière observation vaut aussi bien pour la négation existentielle perfective wèi 'n'y avoir pas eu' dans les exemples (35) et (36) que pour la négation déontique wú 'ne pas devoir' dans l'exemple (37).

(35) 子未我應也。

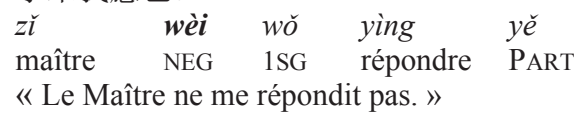

(Mozi,Gong meng)

« Le Maître ne me répondit pas. »

Deux propositions indépendantes peuvent être coordonnées. La coordination peut être formulée par simple juxtaposition (ex. (36) et (37)), explicitée par la présence d'un adverbe réitératif comme yi 'aussi' (ex. (38)) ou celle d'un adverbe argumentatif comme zé 'alors; conséquemment' (ex. (39)) :

(36) 蓋有之矣, 我未之見也

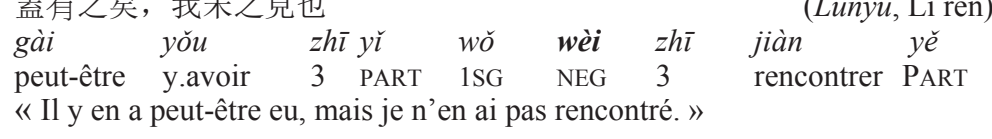

(37) 必交修余, 無余棄也

bi jiāo xiū yú wú yú qì (Guoyu, Chuyu 1)

devoir tous instruire 1SG NEG 1SG abandonner PART

« Vous devez tous m'instruire et ne pas m'abandonner. »

(38) 吾未之樂也亦未之不樂也 (Zhuangzi, Zhi le) wú wèi zhīlè yě yì wèi zhō bù lè yè 1 SG NEG 3 se.réjouir PART aussi NEG 3 NEG se.réjouir PART « Je ne m'en suis réjoui ni ne m'en suis pas réjoui. » 
(39) 戎狄是膺, 荊舒是懲, 則莫我敢承 (Shijing, Lu song, Bi gong) róng dí shì yīng jīng shū shì chéng zé mò wǒ găn chéng Rong Di DEM résister Jing Shu DEM réprimer alors NEG 1SG oser soutenir «Ce sont les barbares Rong et Di auxquels nous résistâmes, les barbares Jing et Shu que nous réprimâmes, si bien que nul (désormais) n'oserait se mesurer à nous. »

La même structure [NEG PRON.OBJ V] se retrouve également dans une proposition principale qui, tout comme une proposition indépendante, se doit de satisfaire aux conditions de finitude. C'est notamment le cas en (40), où la principale est précédée d'une subordonnée concessive, et en (41), où la principale est précédée d'une subordonnée hypothétique :

(40) 以吾一日長乎爾冊吾以也

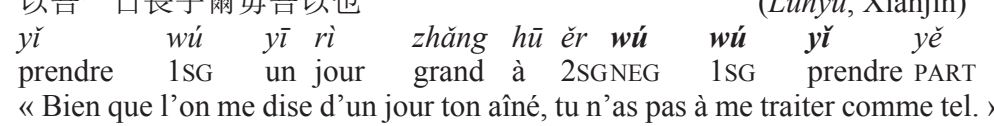

(41) 不克敬于和, 則無我怨

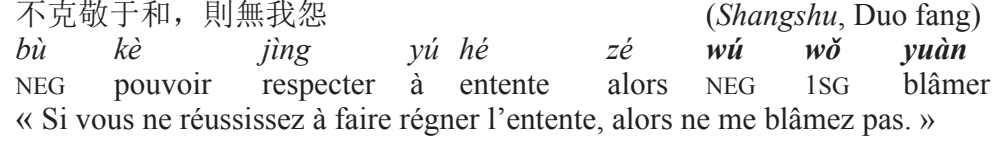

Noter que l'on retrouve cette même structure dans des propositions subordonnées qui satisfont également aux conditions de finitude. Ce phénomène est illustré par l'exemple (42) où la subordonnée concessive, construite avec un véritable subordonnant (suī 'bien que'), arbore l'ordre [NEG PRON.OBJ V] :

$$
\begin{aligned}
& \text { 丘雖不吾譽，吾獨不自知邪? } \\
& q i \bar{u} s u \bar{l} \quad \text { bù wú yù wú dú bù zì zhì yé } \\
& \text { (Zhuangzi, 29.1.19) } \\
& \text { Qiu bien.que NEG 1SG louer 1SG seul NEG soi savoir Q } \\
& \text { «Quand bien même Qiu ne m'adresserait de louanges [pour mes vertus], ne les }
\end{aligned}
$$

\subsubsection{Position postverbale}

Il convient de distinguer trois cas de figure; tous trois renvoient à des contextes non-indépendants et, en termes plus nettement discriminants, à des contextes nonfinis. Le premier cas, illustré par les ex. (43) et (44), concerne les relatives en zhě. ${ }^{3}$ Ce dernier terme joue le rôle de tête nominale et renvoie à une valeur pronominale déictique (='celui qui... ; ceux qui...'). Les verbes des relatives ainsi formées ne sauraient porter de valeur temporelle et correspondraient davantage à nos formes participiales qu'à des verbes finis :

\footnotetext{
${ }^{3}$ Ce phénomène a très tôt été rapporté par Julien (1869: 147), sans explication.
} 
(43) 知我者謂我心懮, 不知我者謂我何求 (Shijing, Wangfeng, Shuli) $\boldsymbol{z h} \overline{\boldsymbol{\imath}} \boldsymbol{w \boldsymbol { c }} \boldsymbol{z h} \check{\boldsymbol{e}}$ wèi wǒ xīn $y \bar{o} u$ connaître $1 \mathrm{SG}$ NMZ dire $1 \mathrm{SG}$ cœur triste

bù $\boldsymbol{z} \boldsymbol{h} \overline{\boldsymbol{u}}$ wǒ $\boldsymbol{z} \boldsymbol{h} \check{\boldsymbol{e}}$ wèi wǒ hé qiú NEG connaitre 1SG NMZ dire $1 \mathrm{SG}$ quoi chercher «Ceux qui me connaissaient demandaient si j'étais triste; ceux qui ne me connaissaient pas demandaient ce que je voulais. » (Lit. : Ceux me connaissant demandaient..., ceux ne me connaissant pas demandaient...)

(44) 弗知之者異于義而同于俗

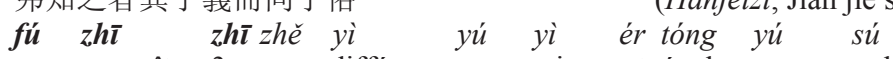

NEG connaître 3 NMZ différer PREP raison et égaler PREP vulgaire

«Ceux ne reconnaissant pas cela s'éloignent de la raison pour adhérer à

l'opinion commune.»

Le deuxième cas figure est celui où l'on a affaire à une proposition adverbiale préverbale, apparaissant entre le sujet (lorsqu'il est présent) et le verbe de la phrase matrice (cf. ex (45)-(46)). Noter que la proposition en question reste non-finie et que son verbe ne saurait ouvertement prendre de sujet (le sujet en tête d'énoncé est syntaxiquement celui du verbe principal) :

(45) 子不與我, 不得歸

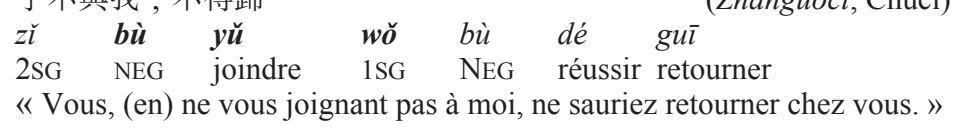

(46) 女嬖大夫而弗下之, 不尊貴也 rǔ bì dàifū ér fú xi⿳亠㐅⿵ zhī bù zūn guì yě 2SG inférieur officier et NEG s'abaisser3 NEG respecter noblesse PART « Vous, en étant officier subalterne et en ne vous abaissant pas devant lui, ne vous montrez pas respectueux de la hiérarchie.»

(47) 無為謂不應我, 非不我應, 不知應我也

\begin{tabular}{|c|c|c|c|c|c|c|}
\hline wú-wéi-wèi & $b \grave{u}$ & yìng & $w \check{o}$ & $f \bar{e} i$ & bù & wŏ \\
\hline Non-agir-discours & NEG & répondre & $1 \mathrm{SG}$ & ne.pas.être & NEG & $1 \mathrm{SG}$ \\
\hline yìng & $z h \bar{\imath}$ & ying & wǒ & $y \check{e}$ & & \\
\hline répondre NEG & savoir & répondre & $1 \mathrm{SG}$ & PART & & \\
\hline
\end{tabular}

Ce dernier exemple est constitué d'une seule et même phrase où les trois propositions arborent successivement les ordres [NEG V PRON.OBJ], [NeG PrOn.OBJ V] et [NEG V PrON.OBJ]. Nous avons tout d'abord une proposition

${ }^{4}$ Lit. 'Discours Non Agissant' est ici une personnification, procédé fréquent chez Zhuangzi. 
adverbiale non finie (bù yìng wǒ 不應我 'en ne me répondant pas') où le pronom objet apparaît en position postverbale; nous avons ensuite un premier contexte matrice où le pronom objet précède le verbe fini ; enfin, un deuxième verbe matrice coordonné (bù zhī yìng wǒ yě 不知應我也 'mais il n'a pas su me répondre') où le pronom objet apparaît en position postverbale car il reste hors de portée de la négation bù qui n'affecte que le verbe principal $z h \bar{l}$ 知 'savoir'.

Le troisième et dernier cas de figure est celui où l'on a affaire à un topique propositionnel nominalisé. Il se distingue $1^{\circ}$ d'une subordonnée en ce qu'il ne prend aucune marque de subordination (telle que suì 'quoique', rú 'si' etc.) ; $2^{\circ}$ d'une proposition adverbiale en ce qu'il peut prendre un sujet; $3^{\circ}$ d'une proposition indépendante en ce qu'il peut être clos par la particule de topicalisation yě (cf. ex. (50)). On notera cependant que le lien entre le topique propositionnel et la phrase matrice qui le suit peut donner lieu à diverses réinterprétations d'ordre pragmatique : hypothétique (ex. (48) et (49)), causale (ex. (50)), temporelle etc.

(48) 爾之許我我其以璧與珪歸俟爾命爾不許我, 我乃屏璧與珪

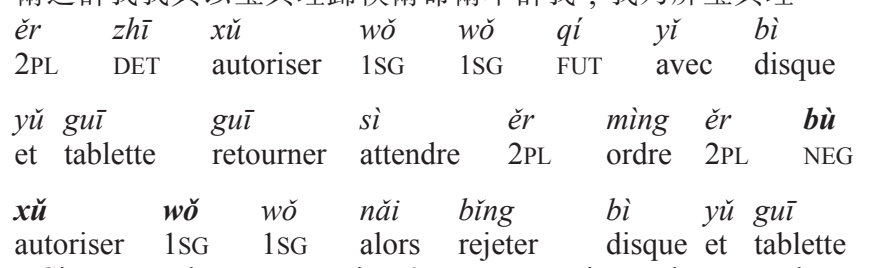

« Si vous me le permettez, je m'en retournerai attendre vos ordres avec en mes mains disque et tablette de jade ${ }^{5}$; si vous ne me le permettez pas, je jetterai alors disque et tablette de jade. »

(Lit. '(Considérant) votre permission à mon égard, je...; vous, ne me le permettant pas, je...')

(Shangshu, Jinteng)

Dans l'exemple ci-dessus, il convient de souligner le parallèle entre le topique propositionnel qui nous intéresse ici, à savoir ěr bù xǔ wǒ [2PL NEG autoriser 1SG], et sa contrepartie affirmative explicitement nominalisée en tout début d'énoncé à l'aide de la marque de détermination $z h \bar{l}$, à savoir ěr zhī xǔ wǒ [2PL DET autoriser 1SG] (lit. 'La vôtre permission à mon égard'). Ce parallèle impose une interprétation non-prédicative des verbes présents dans ces séquences, confortant s'il le faut l'analyse de ces dernières en tant que topique.

(49)

$$
\begin{aligned}
& \text { 曰：有事而不告我, 必不捷矣。 } \\
& \text { yuē yǒu shì ér bù gào wo bì } \\
& \text { dire y.avoir fait et NEG annoncer 1SG nécessairement } \\
& \text { bù jié } y \check{\imath} \\
& \text { « Il dit : «S'il advient quelque fait et que vous ne m'en informiez pas, il est }
\end{aligned}
$$

\footnotetext{
${ }^{5}$ Il s'agit là d'objets symboliques du pouvoir royal.
} 
certain que nous ne réussirons pas. » (Lit. « La survenance d'un fait et la non information de ma personne, c'est nécessairement ne pas réussir »).

(50)

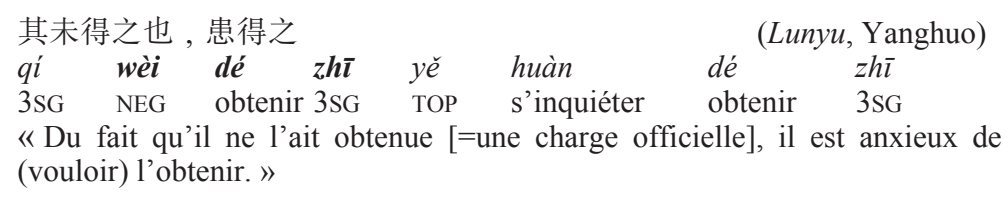

On ne manquera pas de relever dans ce dernier exemple la présence de la marque explicite de topicalisation yě (à ne pas confondre avec la particule finale homophone (cf. ex. (46) et (47)) et celle du pronom possessif de troisième personne qí. Ce dernier révèle clairement le caractère nominal de la proposition topique (Lit. 'Sa non-obtention de cela...').

\section{CONCLUSION}

Nous avons montré que la contrainte dans une phrase négative pour un pronom objet d'apparaître en position préverbale [(S) NEG PRON.OBJ V] est observable dès les inscriptions Shang $\left(13^{\mathrm{e}}-11^{\mathrm{e}} \mathrm{s}\right.$. av. J.-C.). Seul l'usage de la négation bù est alors permis. Cette négation, qui fonctionne par ailleurs comme copule verbale, permet d'extraire le pronom objet et de former ainsi une phrase clivée. Il est à noter que ce clivage n'induit plus la lecture focalisante dont témoignent encore des structures analogues dans les mêmes documents (notamment avec l'emploi des copules wéi ou hui). Force est de constater par ailleurs que la séquence [bù PRON.OBJ] correspond de fait à un ordre VO. On retrouve là l'ordre fondamental qui, aujourd'hui encore, caractérise le chinois (cf. Hagège 1985) ; il ne s'agit en aucun cas d'un reliquat OV d'une langue proto-sinitique qui serait passée, dès l'époque historique, de SOV à VSO (contra Li \& Thompson 1974).

Le manque de document ne permet malheureusement pas de retracer l'évolution précise de cette structure entre le $11^{\mathrm{e}}$ et le $8^{\mathrm{e}} \mathrm{s}$. av. J.-C. On constatera néanmoins qu'entre le $8^{\mathrm{e}}$ et le $2^{\mathrm{e}} \mathrm{s}$. av. J.-C. la contrainte pour les pronoms-objets d'apparaître en position préverbale dans les phrases négatives s'est généralisée à l'ensemble des négations. Cependant, cette contrainte s'est appliquée avec plus ou moins de rigueur selon la nature du pronom-objet et celle de la négation.

Il est intéressant de constater que les pronoms-objets de 1ère et $2 \mathrm{e}$ personne, quelle que soit la négation, apparaissent en position préverbale dans les propositions finies, qu'elles soient principales, indépendantes ou subordonnées. En revanche, dans les propositions dépendantes non-finies (proposition nominalisée, topique propositionnel, proposition adverbiale), ils apparaissent en position postverbale.

\section{BIBLIOGRAPHIE}

Aldridge E., 2015, Pronominal object shift in Archaic Chinese, in T. Biberauer and G. Walkden (eds.), Lexical, Morphological, and Information-Structural 
Interactions, Oxford, Oxford University Press, p. 350-370.

Boodberg P., 1937, Some proleptical remarks on the evolution of Archaic Chinese, Harvard Journal of Asian Studies 2, p. 329-372.

Chen Mengjia, 1956, Yinxu buci zongshu [Etudes sur les inscriptions divinatoires du site archéologique des Yin], Beijing, Kexue chubanshe.

Djamouri R., 1988, Etude des formes syntaxiques dans les écrits oraculaires gravés sur os et écaille de tortue (Chine $14^{e}-11^{e} a v$. J.-C.). Thèse de doctorat, Ecole des Hautes Etudes en Sciences Sociales, Paris.

Djamouri R., 1991, Particules de négation dans les inscriptions sur bronze de la dynastie des Zhou, Cahiers de Linguistique Asie Orientale 20/1, p. 5-76.

Djamouri R., 2000, Preverbal position of the pronominal object in Archaic Chinese, Paper presented at the 9th International Conference on Chinese Linguistics, The National University of Singapore.

Djamouri R., 2001, Markers of predication in Shang bone inscriptions, in Hilary Chappell (ed.), Sinitic Grammar. Synchronic and diachronic perspectives, Oxford et alibi, Oxford University Press, p. 143-171

Djamouri R., 2014, Dui shanggu hanyu foudingju li daici binyu weizhi de jin yi bu taolun [Remarques concernant la position du pronom objet dans la phrase négative en chinois archaïque], Lishi yuyanxue yanjiu 8, p. 47-57.

Djamouri R., Paul W., 1997, Les syntagmes prépositionnels en yu et zai en chinois archaïque, Cahiers de Linguistique Asie Orientale 26/2, p. 221-248.

Djamouri R., Paul, W., Whitman J., 2013, Syntactic change in Chinese and argument-adjunct asymmetry, in Cao G., H. Chappell, R. Djamouri \& T. Wiebusch (eds.), Breaking down the barriers : Interdisciplinary studies in Chinese linguistics and beyond, Taipei, Institute of Linguistics, Academia Sinica [Language and Linguistics Monograph Series 50], p. 557-594.

Dobson W.A.C.H., 1960, Early Archaic Chinese, Toronto, University of Toronto Press.

Gao Mingkai, 1946, A Study of Personal Pronouns in the Chinese Language, Yenching Journal 30, p. 1-26.

Graham A. C., 1952, A Probable Fusion-word: $w u h=w u+j y$, Bulletin of the School of Oriental and African Studies XIV/I, p. 139-148.

Guan Xiechu, 1953, Yinxu jiagu keci de yufa yanjiu [Etude grammaticale des inscriptions sur os et carapaces du site archéologique des Yin], Shanghai, Guojia kexue.

Hagège C., 1975, Le problème linguistique des prépositions et la solution chinoise (avec un essai de typologie à travers plusieurs groupes de langues), Paris, Société de Linguistique de Paris.

Hagège C., 1985, Le chinois, l'ordre des mots et l'ordre du pensable, Toun'g Pao LXXI, p. 263-274.

He Leshi, 2001, 'Fu' de lishi yanbian [Evolution historique de $f u$ ], in R. Djamouri (ed.) Collected essays in Ancient Chinese Grammar, Paris, EHESS-CRLAO, p. 81-98

Julien S., 1869, Syntaxe nouvelle de la langue chinoise fondée sur la position des mots, Paris, Librairie de Maisonneuve.

Kennedy D. N., 1952, Negatives in Classical Chinese, Wennti 1, p. 1-16. 
Kong Yingda (574-648), Mao shi zhengyi. (réimp. Beijing, Beijing daxue chubanshe)

Kryukov M. V., 1980, The language of Yin inscriptions, Moscow, NAUKA publishing house.

Li C. N. and S. A. Thompson, 1974, An explanation of word order change SVO > SOV, Foundations of Language 12, p. 201-214.

Lü Shuxiang, 1942 (réimp. 1956), Zhongguo wenfa yaolüe [Précis de grammaire chinoise], Shanghai, Shangwu yinshuguan.

Ma Jianzhong, 1898. Mashi wentong, Shanghai, Shangwu yinshuguan.

Meisterernst B., 2010, Object Preposing in Classical and Pre-Medieval Chinese, Journal of East Asian Linguistics 19, p. 75-102.

Mulder J., 1959, On the Morphology of the Negatives in Archaic Chinese, T'oung Pao XLVII/3-5, p. 251-280.

Onishi K., 1992, Yin zhou shidai di yi rencheng daimingci de yongfa yanjiu [Etude sur l'emploi des pronoms personnels de première personne aux époques $\mathrm{Y}$ in et Zhou], Chugoku gogaku 239, p. 115-124.

Pan Yunzhong, 1982, Hanyu yufa shi gaiyao [Précis de grammaire historique du chinois], Henan, Zhongzhou shuhuashe.

Peyraube A., 1997, On word order and word order change in pre-Archaic Chinese, in Symposium Series of the Institute of History and Philology Academia Sinica 2, p. $105-124$.

Rémusat A., 1857, Elémens de la grammaire chinoise ou principes généraux de Kou-wen ou style antique et du Kouan-hoa, c'est-à-dire, de la langue généralement usitée dans l'empire chinois, Paris, Maisonneuve et $\mathrm{C}^{\mathrm{ie}}$.

Serruys P.L.M., 1974, Studies of the Language of the Shang Oracle Inscriptions, T'oung Pao LX/1-3, p. 12-120.

Serruys P.L.M., 1981, Towards a Grammar of Shang Bone Inscriptions, in Zhongyang yanjiuyuan guoji hanxue huiyi lunwenji, Taipei, Zhongyang yanjiuyuan yuyan wenzi zu, p. 313-364.

Shen Pei, 1992. Yinxu jiagu buci yuxu yanjiu, Taipei, Wenjin.

Takashima K., 1990, A Study of the Copulas in Shang Chinese, The Memoirs of the Institute of Oriental Culture (University of Tokyo) 112, p. 1-92.

Takashima K., 1973, Negatives in the King Wu-Ting Bone Inscriptions, Ph.D. dissertation, Seattle, University of Washington.

Takashima K., 1988, Morphology of the Negatives in Oracle-Bone Inscriptions. Computational Analyses of Asian and African Languages 30, p. 113-133.

Tang Yuming, 1990, Jiaguwen 'wei-bin-dong' shi jiqi tuibian [Construction 'wei$\mathrm{O}-\mathrm{V}$ ' dans les jiaguwen et son évolution], Zhongshan daxue xuebao 3, 120$126+133$.

Von Der Gabelentz G. 1881 Chinesische Grammatik mit Ausschluss des niederen Stiles und der heutigen Umgangsprache. Leipzig, T. O. Weigel.

Wang Li, 1958, Hanyu shi gao [Ebauche d'histoire de la langue chinoise], Beijing, Kexue chubanshe.

Zhang Yujin, 2001, Jiaguwen yufaxue [Etude grammaticale des jiaguwen], Shanghai, Xuelin chubanshe.

Zhou Fagao, 1972, Zhongguo gudai yufa - chengdai bian [Grammaire ancienne 
du chinois - partie sur les pronoms], Taipei, Tailian guofeng.

Zhou Guangwu, 1959, Xianqin fouding ju daici binyu weizhi wenti [Question de la position du pronom objet dans les phrases négatives à l'époque pré-Qin], in Zhongguo yuwen zazhi she (ed.), Yuwen lunji 3, Beijing, Zhonghua shuju, p. 128-192.

Zhou Shengya, 1980, Lun shanggu hanyu rencheng daici fanfu de yuanyin [Examen des raisons de l'existence de nombreux pronoms personnels en chinois archaïque], Zhongguo yuwen 2, p. 127-136. 